Check for updates

Cite this: Chem. Commun., 2020, 56, 2929

Received 26th November 2019, Accepted 20th December 2019

DOI: $10.1039 / \mathrm{c} 9 \mathrm{cc} 09204 \mathrm{~h}$

rsc.li/chemcomm

\title{
Degrasyn exhibits antibiotic activity against multi-resistant Staphylococcus aureus by modifying several essential cysteines $\dagger$
}

\author{
Kyu Myung Lee, (D) ${ }^{a}$ Philipp Le, ${ }^{a}$ Stephan A. Sieber (D)*a and Stephan M. Hacker (D) *b
}

\begin{abstract}
Degrasyn inhibits deubiquitination enzymes and has anti-cancer activity. We here show that it also exhibits antimicrobial activity against multiresistant Staphylococcus aureus. Structure activity relationship studies demonstrate an important role of the electrophilic $\alpha$-cyanoacrylamide moiety as a Michael acceptor. A suite of chemical proteomic techniques unraveled binding of this moiety to various cysteine residues of essential proteins in a reversibly covalent manner.
\end{abstract}

Rapid bacterial resistance development represents a major challenge for current antibacterial therapies. ${ }^{1,2}$ One strategy to identify novel antibacterials focuses on the repurposing of existing drugs originally developed against human proteins. ${ }^{3,4}$ In a recent drug-repurposing screen, ${ }^{5}$ we identified that the human deubiquitinating enzyme inhibitor degrasyn (also called WP1130) kills methicillin-sensitive S. aureus (MSSA) (Fig. 1A and B). Degrasyn induces apoptosis of leukemia cells. ${ }^{6,7}$ In addition, anti-infective effects, i.e. the reduction of intracellular replication of Listeria monocytogenes and viruses, were previously reported. These effects were mainly attributed to the inhibition of DUBs in macrophages. ${ }^{8-10}$ Direct antibiotic effects of degrasyn on isolated bacteria have to the best of our knowledge not been reported so far.

Here, we synthesized 19 degrasyn derivatives and analyzed their effects on bacterial growth. The nitrile-substituted Michael acceptor ( $\alpha$-cyanoacrylamide) turned out to be a signature moiety important for antibiotic activity through reversible covalent modification of cysteine residues in essential enzymes. Chemical proteomics revealed several target proteins of degrasyn belonging to important enzyme classes involved e.g. in cell wall, lipid and histidine biosynthesis indicating a polypharmacological mode-ofaction.

\footnotetext{
${ }^{a}$ Center for Integrated Protein Science, Department of Chemistry and Chair of Organic Chemistry II, Technische Universität München, Garching bei München, Germany.E-mail: stephan.sieber@tum.de

${ }^{b}$ Department of Chemistry, Technische Universität München, Garching bei München, Germany. E-mail: stephan.m.hacker@tum.de

$\dagger$ Electronic supplementary information (ESI) available: Supporting scheme, figures and tables, experimental procedures and compound characterization. See DOI: $10.1039 / \mathrm{c} 9 \mathrm{cc} 09204 \mathrm{~h}$
}

Given the promising antibiotic effects of degrasyn against the MSSA strain NCTC8325 with a minimal inhibitory concentration (MIC) of $6.25 \mu \mathrm{M}$, we tested its activity against various methicillin-resistant $S$. aureus (MRSA) strains including several clinical isolates (Fig. 1B)..$^{5,11}$ Satisfyingly, the antibiotic activity was retained in all MRSA strains (MIC $\leq 12.5 \mu \mathrm{M}$ ) suggesting a different mode-of-action as compared to existing drugs.

To elucidate the structure activity relationships (SAR) of degrasyn, we systematically altered its scaffold. Degrasyn (DGS) and its enantiomer $((\boldsymbol{R})$-DGS) were prepared according to established synthetic procedures (Scheme 1). A change of the stereocenter resulted in a slight drop of the MIC from $6.25 \mu \mathrm{M}$ for DGS to $12.5 \mu \mathrm{M}$ for $(\boldsymbol{R})$-DGS suggesting only a minor role of the absolute configuration (Fig. 1C). Accordingly, a racemic mixture of $(\boldsymbol{R}, \boldsymbol{S})$-DGS exhibited the same MIC value as DGS. Due to the minor role of the stereocenter and for the ease of synthesis, all further products were synthesized as racemic mixtures.

The synthesis of DGS variants with modifications at the pyridine ring (1-15, Fig. 1C) followed a modular blueprint as outlined in Scheme 2. While an unsubstituted pyridine ring led to a loss of antibiotic activity $(\mathbf{1}, \mathbf{2})$, varying the positions of the bromine substituent and the nitrogen atom was tolerated with only a slight loss in activity $(3,4)$. Even the replacement of the pyridine ring with a phenyl ring retained considerable activity (5). Therefore, due to availability of building blocks and synthetic accessibility, we studied substituted phenyl rings at this position in more detail. Bromine- and chlorine-substituted phenyl rings were able to largely maintain antibiotic effects $(\mathbf{5}, \mathbf{6}, \mathbf{7})$. In contrast, fluoro- and iodo-substituted phenyl rings resulted in a loss of activity $(8,9,10)$. While di- and tri-substitutions with electrondonating groups (e.g. - $\mathrm{OH},-\mathrm{OMe}$, etc.) were largely not tolerated $(11,12,13)$, the introduction of a difluoroacetal moiety $(14)$ retained full activity. Satisfyingly, substitution with an alkyne handle (15) only slightly increased the MIC, which is a prerequisite for subsequent target identification using conventional ABPP.

We next turned our synthetic efforts towards the reactive $\alpha$-cyanoacrylamide moiety. Acrylamides are irreversible covalent inhibitors, which readily react with cysteine residues. ${ }^{12}$ Further 
A)

C)
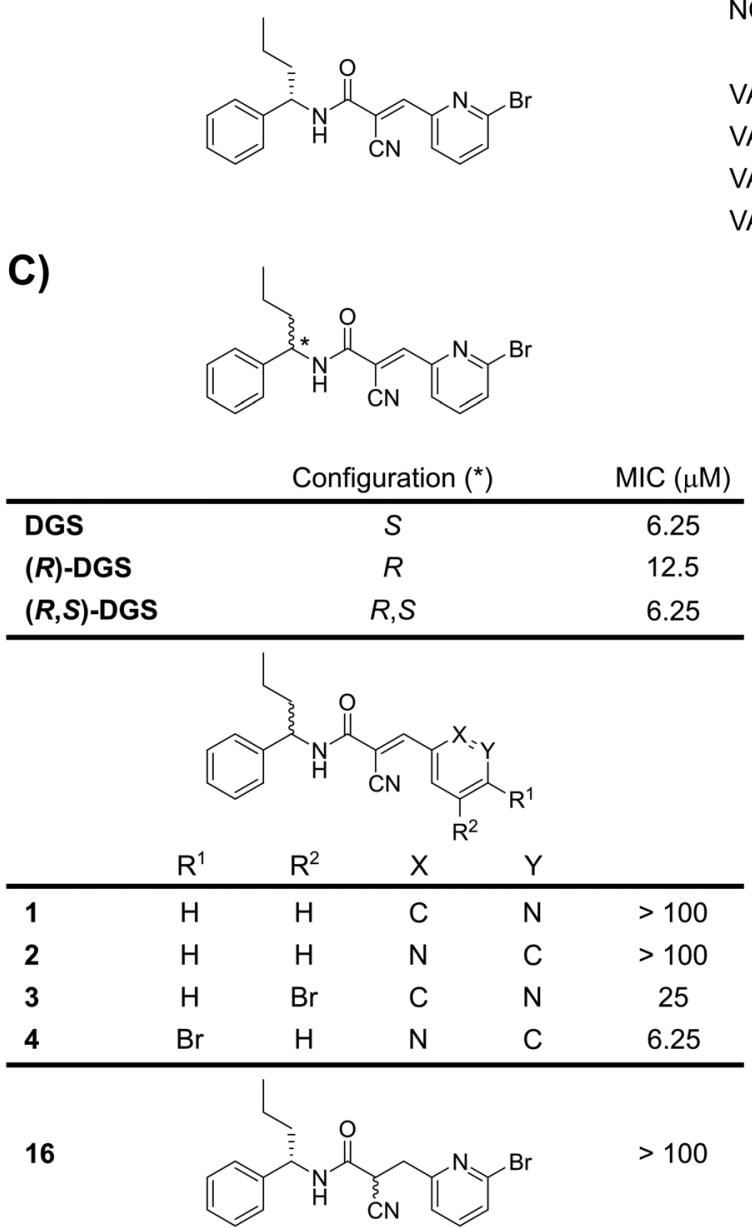

B)

\begin{tabular}{cc|cc} 
Strain & MIC $(\mu \mathrm{M})$ & Strain & MIC $(\mu \mathrm{M})$ \\
\hline NCTC8325 & 6.25 & VA418879* & 6.25 \\
MU50 & 12.5 & VA417350* & 12.5 \\
VA402525* & 12.5 & IS050611* & 12.5 \\
VA409044* $^{*}$ & 12.5 & IS050678* & 12.5 \\
VA402923* & 6.25 & BK097296* & 6.25 \\
VA412350* & 12.5 & BK095395* & 6.25
\end{tabular}<smiles>[R]c1cc(/C=C(\C)C(=O)N[C@@H](CCC)c2ccccc2)c([R])c([R])c1[R]</smiles>

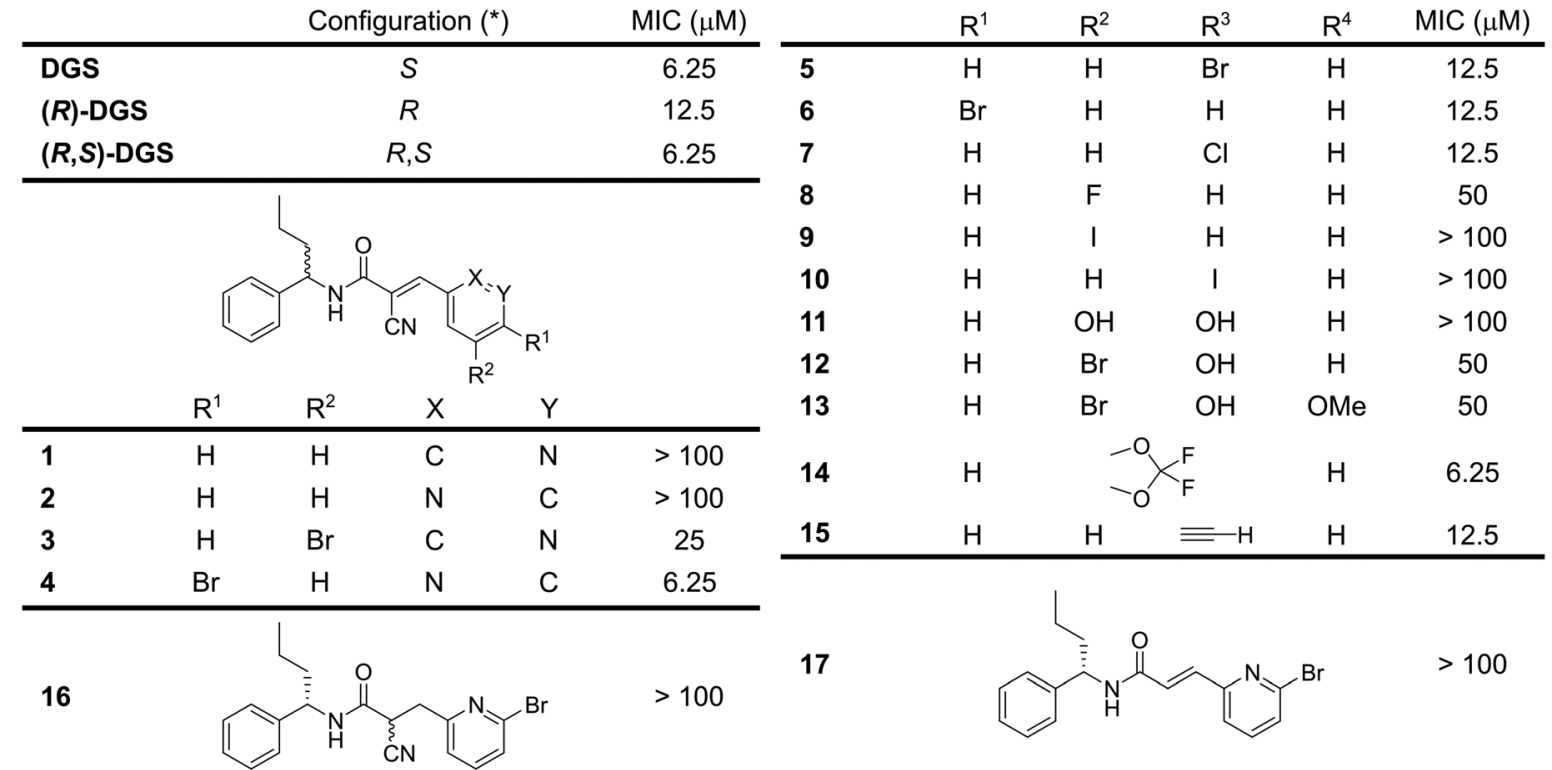

Fig. 1 (A) Structure of degrasyn (DGS). (B) MIC values for DGS in various strains of S. aureus. *: Clinical isolates of S. aureus. (C) Substitution patterns and MIC values in S. aureus NCTC8325 for various degrasyn analogues synthesized in this study in order to investigate the SAR of DGS

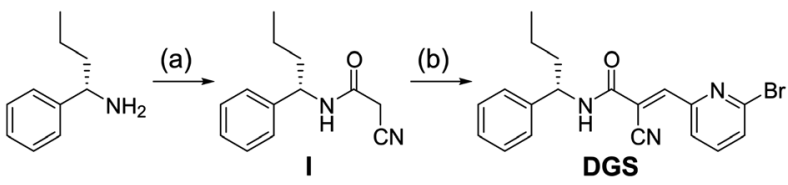

Scheme 1 Synthesis of DGS. (R)-DGS was synthesized using the same protocol starting from (R)-1-phenylbutan-1-amine. (a) 1-Cyanoacetyl-3,5-dimethyl$1 \mathrm{H}$-pyrazole, toluene, reflux, 2 h, 75\%; (b) 6-bromo-2-pyridinecarboxaldehyde, piperidine, $\mathrm{EtOH}$, reflux, $3 \mathrm{~h}, 65 \%$.

fine-tuning with electron-withdrawing substituents such as nitriles yields reversibly covalent inhibitors, which have received great attention in drug development. ${ }^{13,14}$ As degrasyn bears this signature moiety, we investigated, if the ability to act as a Michael acceptor and the fine-tuning as $\alpha$-cyanoacrylamide are both relevant for the antibiotic effect. The synthesis of 16, without the double bond of the Michael acceptor, was achieved by reduction of DGS using $\mathrm{NaBH}_{4}$ (Scheme S1, ESI $\dagger$ ). The synthesis of $\mathbf{1 7}$, without the nitrile moiety, was performed using a Horner-Wadsworth-Emmons reaction (Scheme S1, ESI $\dagger$ ). Interestingly, neither 16 nor $\mathbf{1 7}$ showed any

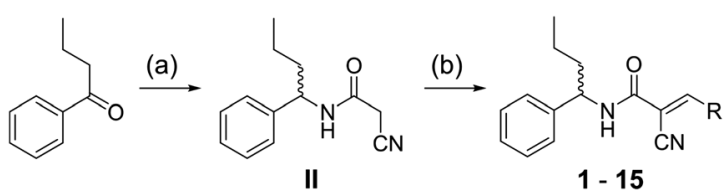

Scheme 2 Synthesis of degrasyn derivatives (see Fig. 1 for specific $\mathrm{R}$ groups). (a) (i) $\mathrm{NH}_{2} \mathrm{OH} \cdot \mathrm{HCl}, \mathrm{NaOH}, \mathrm{EtOH} / \mathrm{H}_{2} \mathrm{O}, 3 \mathrm{~h}$ (ii) $\mathrm{H}_{2}, \mathrm{Pd} / \mathrm{C}, \mathrm{MeOH}$, $24 \mathrm{~h}$ (iii) 1-cyanoacetyl-3,5-dimethyl- $1 \mathrm{H}$-pyrazole, toluene, reflux, $2 \mathrm{~h}, 69 \%$; (b) aryl aldehyde, piperidine, EtOH, reflux, 3 h, 33-75\%.

antibiotic activity highlighting that both the Michael acceptor and the electron-withdrawing substituent are mandatory (Fig. 1C).

In order to better understand the mechanism-of-action, we performed target identification studies in $S$. aureus. We first applied conventional ABPP with 15 (Fig. S1, ESI $\dagger$ ). ${ }^{15,16}$ Live bacterial cells were incubated with the probe, lysed, labeled proteins modified with biotin azide using copper-catalyzed azide-alkyne cycloaddition (CuAAC) ${ }^{17}$ and subsequently analyzed via liquid chromatography coupled to tandem mass spectrometry (LC-MS/MS). The labeling was performed at three different 
concentrations $(6.25 \mu \mathrm{M}, 12.5 \mu \mathrm{M}$ and $25 \mu \mathrm{M})$ and data analysis was performed with label-free quantification (LFQ). ${ }^{18}$ While we saw some labeling in a gel-based analysis, no prominent enrichment of protein targets was observed by mass spectrometry (Fig. S2 and Table S1, ESI $\dagger$ ). In addition, competition studies with various concentrations of parent DGS did not lead to an obvious target profile (Fig. S3 and Table S1, ESI $\dagger$ ). Thus, the reversibly covalent binding mechanism is not suitable for conventional ABPP studies as the transient target binding is incompatible with the enrichment procedure for MS.

We therefore performed competitive residue-specific proteomics using the recently developed isoDTB-ABPP method ${ }^{19}$ that is based on the isoTOP-ABPP (isotopic tandem orthogonal proteolysis-ABPP) platform $^{12,20}$ as an alternative strategy. In this approach, S. aureus cells are lysed and the lysate is split into two samples. One sample is treated with DGS and the other one with DMSO as a control (Fig. 2A). Afterwards, the samples are both alkynylated with iodoacetamidealkyne (IA-alkyne). Competitive cysteine binders such as DGS block this labeling at their specific binding sites in the proteome. In order to read out these differences in alkynylation, isotopically labeled desthiobiotin azide (isoDTB) tags ${ }^{19}$ are appended using CuAAC. ${ }^{17}$ The samples are combined, enriched on streptavidin, and digested. The resulting peptides are identified and quantified relative to each other using LC-MS/MS. The ratio $R$ between the heavy-labeled (DMSO-treated) and light-labeled (DGS-treated) samples is a measure for the degree of modification of the specific cysteine with DGS.
Besides the ability to obtain residue-specific information of binding with an unmodified covalent protein ligand, another important advantage of this approach is that also cysteines, which are reversibly engaged by DGS, are identified. ${ }^{14}$ Satisfyingly, treatment of $S$. aureus lysate with various concentrations of DGS ranging from $100 \mu \mathrm{M}$ to $10 \mu \mathrm{M}$ led to the identification of 151 cysteines in 114 proteins targeted by DGS (Fig. 2B, Fig. S4 and Table S1, ESI $\dagger$ ). Interestingly, 96 of these cysteines were not liganded by any of 19 covalent $\alpha$-chloro-acetamides that were previously used to profile cysteines in S. aureus. ${ }^{19}$ This indicates that the reversibly covalent $\alpha$-cyano-acrylamide is able to interact with a unique set of cysteines. Many cysteines respond to DGS treatment in a clearly concentration-dependent manner (Fig. 2C and Tables S1, S2, ESI $\dagger$ ). For the 98 cysteines that are liganded by DGS and quantified at all concentrations, the data could be fitted with a dose-response curve with a median $R^{2}$ value of 0.92 . This demonstrates that residue-specific proteomics with the isoDTB tags using a few different concentrations of a covalent protein ligand is able to deliver robust information on the binding affinity for many cysteines in parallel.

31 of the targeted cysteines belong to proteins with an essential function for the viability of $S$. aureus. DGS e.g. binds cysteine $\mathrm{C} 100$ of the bifunctional protein $\operatorname{GlmU}\left(\mathrm{EC}_{50}=22.3 \mu \mathrm{M}\right.$, UniProt Code Q2G0S3), which is located close to the magnesium ion in the UDP-GlcNAc binding site of this protein. ${ }^{21} \mathrm{GlmU}$ is an essential protein in the synthesis of UDP-GlcNAc and therefore

A) isoDTB-ABPP: reversible binding information conserved by IA-alkyne
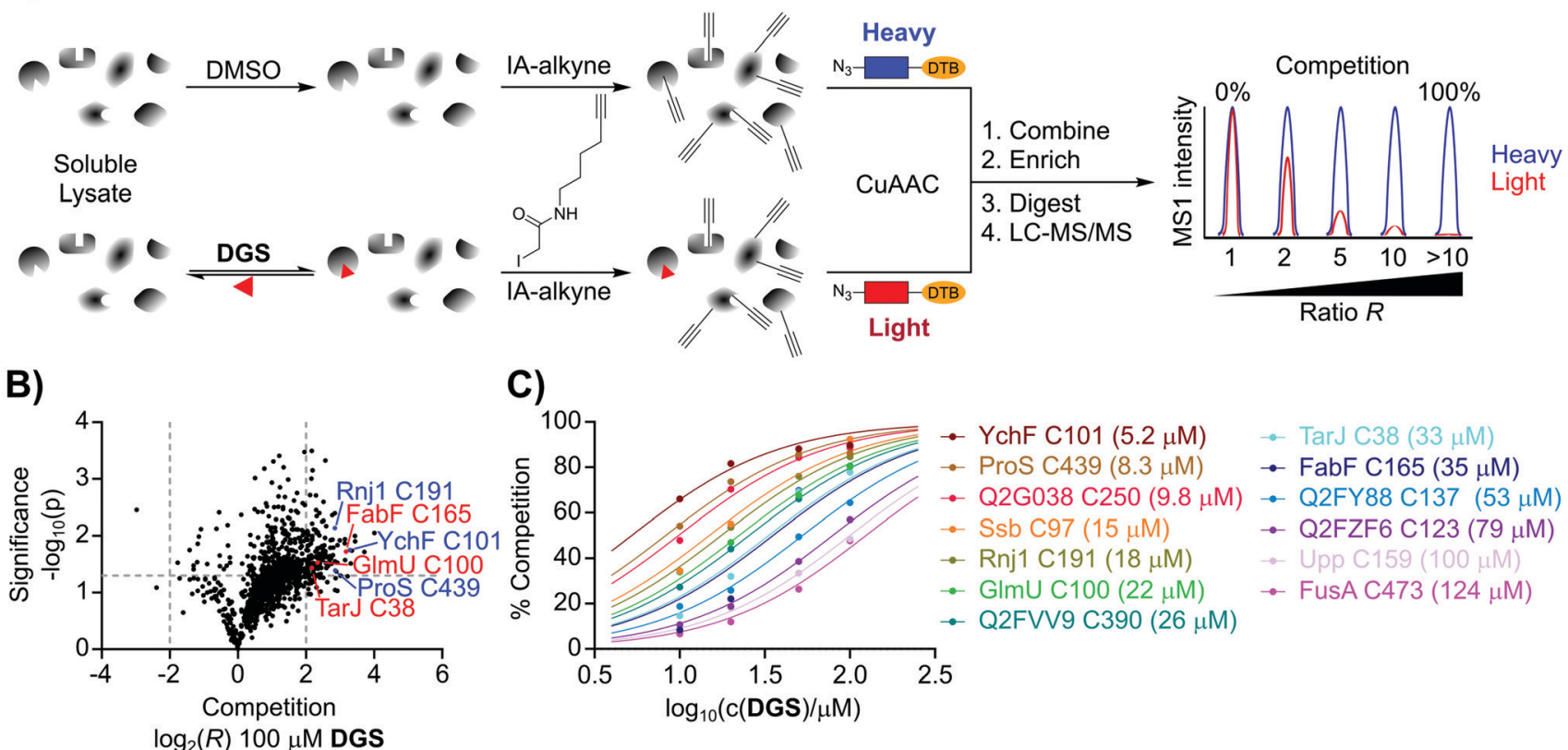

Fig. 2 (A) Workflow for competitive, residue-specific proteomics with the isoDTB-ABPP technology. Competition of IA-alkyne labeling by reversibly binding DGS is preserved throughout the workflow by the irreversibly binding probe. DTB: desthiobiotin. (B) Volcano plot for the isoDTB-ABPP experiment with $100 \mu$ M DGS used as the competitor. Significantly competed cysteines that are discussed in the text are highlighted in red. Other liganded cysteines that are included in the concentration-dependent analysis (C) are highlighted in blue. The grey lines indicate cut-offs at $-\log _{10}(p)=1.3$ and $\log _{2}(R)= \pm 2$ that were used as a criterion for hit selection. (C) Dependence of the degree of competition in isoDTB-ABPP experiments on the DGS concentration. Data points are measured values from isoDTB-ABPP experiments and lines are non-linear dose-response curve fits. The values in parentheses are the $E_{50}$ values of the competition for the respective cysteine. $E_{50}$ values with confidence intervals are given in Table S2 (ESI $\dagger$ ). In (B) and (C), for each indicated cysteine, the name or the UniProt code of the respective protein and the residue number of the competed cysteine are given. All data results from duplicates. 
A)

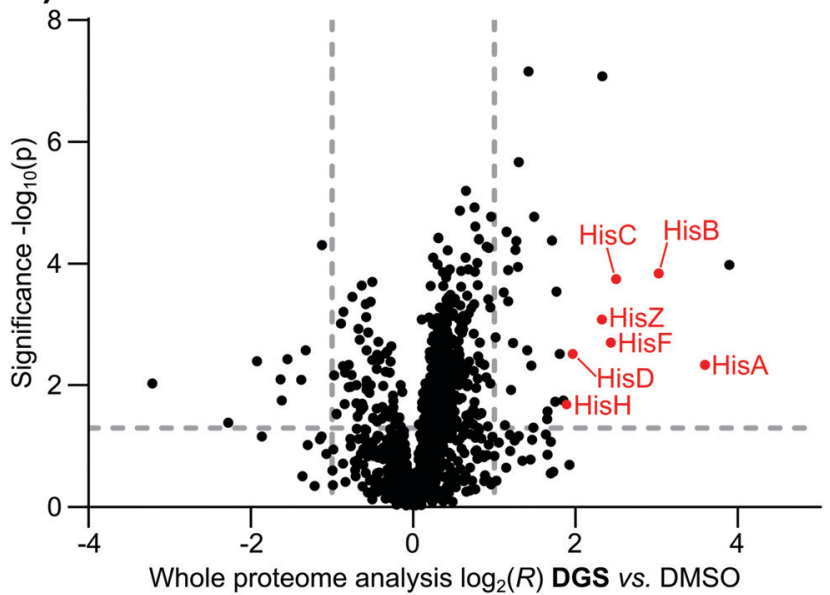

B)

\begin{tabular}{ll} 
Gene Ontology term (biological process) & corr. p-value \\
\hline Histidine biosynthetic process & $3.16 \mathrm{E}-07$ \\
Imidazole-containing compound metabolic process & $3.27 \mathrm{E}-06$ \\
Histidine metabolic process & $3.27 \mathrm{E}-06$ \\
Aromatic amino acid family biosynthetic process & $3.05 \mathrm{E}-05$ \\
Aromatic amino acid family metabolic process & $1.36 \mathrm{E}-04$
\end{tabular}

Fig. 3 (A) Volcano plot for a whole proteome analysis of protein expression comparing S. aureus NCTC 8325 treated with $\frac{1}{2}$ MIC concentration of DGS to treatment with DMSO as a control. Upregulated proteins involved in the histidine biosynthetic process are highlighted in red. (B) Enrichment analysis of Gene Ontology terms of the category "biological process" comparing the upregulated proteins to all proteins detected in the whole proteome analysis. The five terms with the lowest corrected $p$-value are shown. All data results from four independent biological replicates. The grey lines indicate cut-offs at $-\log _{10}(p)=1.3$ and $\log _{2}(R)= \pm 1$ that were used as a criterion for selection of up- and down-regulated proteins.

important in e.g. cell wall biosynthesis. ${ }^{22}$ DGS also binds cysteine C38 of the ribulose-5-phosphate reductase TarJ $\left(\mathrm{EC}_{50}=33.4 \mu \mathrm{M}\right.$, UniProt Code Q2G1B9), which is involved in the binding of the catalytic zinc ion. ${ }^{21}$ TarJ is essential in the synthesis of poly(ribitol phosphate)teichoic acid, which are the main cell wall teichoic acids of $S$. aureus. ${ }^{23}$ Furthermore, modification of the catalytic nucleophile C165 of the 3-oxoacyl-[acyl-carrier-protein] synthase FabF $\left(\mathrm{EC}_{50}=35.3 \mu \mathrm{M}\right.$, UniProt Code Q2FZR9), an enzyme essential for lipid biosynthesis, was detected. ${ }^{21,24}$ In this way, interaction of DGS with cysteine residues in functionally relevant binding sites of several essential proteins could be a major driver of its antibiotic activity.

To further complement these results, we performed a global analysis of cellular protein levels in response to DGS treatment. Cells were cultivated with $\frac{1}{2}$ MIC concentration of DGS (in order to prevent premature cell lysis) and investigated by LC-MS/MS via LFQ. In line with the multiple targets observed by isoDTB-ABPP, a large fraction of 33 proteins was upregulated and only nine proteins were downregulated (Fig. 3A and Table S1, ESI $\dagger$ ). Of the dysregulated proteins seven are essential for $S$. aureus survival, five of which were down-regulated ribosomal proteins. Analysis of the Gene Ontology terms of the upregulated proteins revealed a strong enrichment of terms associated with histidine biosynthesis (Fig. 3B). Together with the isoDTB-ABPP data these results suggest a polypharmacological mode-of-action for DGS leading to the alteration of multiple essential pathways, which is reflected by complex changes in the proteome.

In summary, we identified degrasyn as a novel antibiotically active compound against $S$. aureus including clinically isolated MRSA strains. In line with the essential role of the $\alpha$-cyanoacrylamide as Michael acceptor covalent capture of target proteins failed due to limited stability of the linkage throughout the chemoproteomic protocol. While reversible covalent inhibitors are very promising, this is one technological drawback. Competitive residue-specific proteomics using the isoDTB-ABPP method turned out as an excellent complementary strategy to identify the targeted cysteines of degrasyn in vitro. 31 of these cysteines were labelled in proteins essential for viability of $S$. aureus highlighting a polypharmacological mode-of-action which was further corroborated by the up- or down-regulation of several proteins in a whole proteome study. While for degrasyn significant human toxicity is a challenge $\left(\mathrm{IC}_{50}=3.4 \pm 0.3 \mu \mathrm{M}\right.$ in an MTT assay in A549 cells), the high antibacterial potency obtained by screening a small compound library encourages the repurposing of other human inhibitors or drugs for exploiting their antibacterial potential.

SAS acknowledges funding by the Center for Integrated Protein Science (CIPSM) and the European Research Council (grant agreement No. 725085, CHEMMINE, ERC consolidator grant), SMH by the Fonds der Chemischen Industrie (Liebig Fellowship) and the TUM Junior Fellow Fund and KML by the Korea Research Institute of Chemical Technology (KRICT).

\section{Conflicts of interest}

There are no conflicts to declare.

\section{Notes and references}

1 A. Cassini, et al., Lancet. Infect. Dis., 2019, 19, 56-66.

2 M. Lakemeyer, W. Zhao, F. A. Mandl, P. Hammann and S. A. Sieber, Angew. Chem., Int. Ed., 2018, 57, 14440-14475.

3 A. Miro-Canturri, R. Ayerbe-Algaba and Y. Smani, Front. Microbiol., 2019, 10, 41.

4 L. Peyclit, S. A. Baron and J. M. Rolain, Front. Cell. Infect. Microbiol., 2019, 9, 193.

5 P. Le, et al., Nat. Chem., DOI: 10.1038/s41557-019-0378-7.

6 L. F. Peterson, et al., Blood, 2015, 125, 3588-3597.

7 G. A. Bartholomeusz, et al., Blood, 2007, 109, 3470-3478.

8 M.-E. Charbonneau, et al., PLoS One, 2014, 9, e104096-e104096.

9 K. M. Burkholder, et al., Infect. Immun., 2011, 79, 4850-4857.

10 K. D. Passalacqua, et al., Antimicrob. Agents Chemother., 2016, 60, 4183-4196.

11 J. Vomacka, et al., Chemistry, 2016, 22, 1622-1630.

12 K. M. Backus, et al., Nature, 2016, 534, 570-574.

13 J. M. Bradshaw, et al., Nat. Chem. Biol., 2015, 11, 525-531.

14 K. Senkane, et al., Angew. Chem., Int. Ed., 2019, 58, 11385-11389.

15 M. J. Evans and B. F. Cravatt, Chem. Rev., 2006, 106, 3279-3301.

16 P. P. Geurink, L. M. Prely, G. A. van der Marel, R. Bischoff and H. S. Overkleeft, Top. Curr. Chem., 2012, 324, 85-113.

17 V. V. Rostovtsev, L. G. Green, V. V. Fokin and K. B. Sharpless, Angew. Chem., Int. Ed., 2002, 41, 2596-2599.

$18 \mathrm{~J}$. Cox, et al., Mol. Cell. Proteomics, 2014, 13, 2513-2526.

19 P. R. A. Zanon, L. Lewald and S. M. Hacker, Angew. Chem., Int. Ed., DOI: 10.1002/anie.201912075.

20 S. M. Hacker, et al., Nat. Chem., 2017, 9, 1181-1190.

21 The UniProt Consortium, Nucleic Acids Res., 2019, 47, D506-D515.

22 M. Wang, et al., RSC Adv., 2017, 7, 13858-13867.

23 E. W. Sewell and E. D. Brown, J. Antibiot., 2014, 67, 43-51.

24 Y. Wang and S. Ma, ChemMedChem, 2013, 8, 1589-1608. 